\title{
Análise de Sistemas de Geração de Energia Elétrica com Base na Pegada Ecológica Modificada
}

\author{
Bruno Silva de Carvalho, brunoscarvalho1990@hotmail.com - IFSULDEMINAS; \\ Carlos Cezar da Silva, carlos.silva@ifs.ifsuldeminas.edu.br - IFSULDEMINAS; \\ Cezar Augusto Frimaio da Silva, cafs_sp@msn.com - UFABC
}

\section{RESUMO}

O presente trabalho determina a Pegada Ecológica modificada e a Capacidade de Carga (Zhao et al., 2004) de três sistemas de geração de energia elétrica com uso de combustíveis renováveis e as compara. Foram estudados os sistemas de Utilização de Resíduos Sólidos Urbanos em um Aterro Sanitário na cidade de São Paulo (Frimaio, 2011), Estação de Tratamento de Efluentes na cidade de Uppsala na Suécia (Bjorklund, et al., 2001) e uma Usina Autônoma localizada em São Paulo (Silva, 2006). Os resultados obtidos apontam que o sistema de Aproveitamento de resíduos apresenta melhor performance com relação ao sistema de Estação de Tratamento de Esgoto a Usina Autônoma.

Palavras chaves: emergia, pegada ecológica, usina autônoma, resíduos, energia, ETE.

\section{Analysis of Systems of Generation of Electric Energy on the Basis of the Modified Ecological Footprint}

\section{ABSTRACT}

The present work determines the modified Ecological Footprint and the Load Capacity (Zhao et al., 2004) of three systems of generation of electric energy with fuel use you renewed it compares and them. The systems Station of Treatment of in the city of Uppsala in Sweden (Bjorklund, et al., 2001) in São Paulo had been studied of Use of Urban Solid Residues in Sanitary Aterro in the city of São Paulo (Frimaio, 2011) Effluent located Independent Plant and, (It hisses, 2006). The gotten results point that the system of Exploitation of residues better presents performance with regard to the system of Station of Treatment of Sewer the Independent Plant.

Key words: emergy, ecological footprint, independent plant, residues, energy, STS

\section{INTRODUÇÃo}

Atualmente, a questão ambiental é discutida em todos os meios de comunicação: jornais, revistas, programas de televisão, rádio, etc. Alguns dos assuntos mais abordados são: a questão do aquecimento global, derretimento de geleiras, catástrofes, mas se quer paramos para pensar o que muitas vezes é a causa desses acontecimentos. Preocupa-se muito em colocar a culpa em quem está a nossa volta, ou em quem polui mais ou gera mais resíduos do que nós, mas esquecemos que as nossas ações de uma forma ou de outra acaba por produzir algum tipo de resíduo, danificando assim o ambiente em que vivemos. Cada ser humano que habita o nosso planeta deixa um "rastro", uma "marca", uma Pegada Ecológica. É interessante que pudéssemos aprender a ver as nossas atitudes individualmente, e não simplesmente as atitudes coletivas.

\section{Pegada Ecológica}

A Pegada Ecológica pode ser comparada ao simples caminhar sobre a areia na praia, quando caminhamos calmamente e prestando a atenção em nossos passos podemos verificar o pequeno rastro que deixamos nela, mas quando começamos a correr ou andar com maior intensidade com movimentos mais bruscos, a nossa pegada será maior e mais profunda. É necessário levar em consideração cada um que estão nessa caminhada, cada um terá uma pegada maior ou menor. É citada na literatura como um indicador de sustentabilidade, a qual tem como proposta a quantificação das áreas necessárias para produzir recursos e assimilar os resíduos gerados por certa população (Wackernagel e Rees, 1994).

\section{Pegada Ecológica Modificada}

Utilizando a síntese em emergia Odum, (1996), Zhao et al. (2004) propõem uma cálculo de Pegada Ecológica (PE) e Capacidade de Carga (Cc) modificadas. Assim, a Pegada Ecológica modificada é considerada como 
a área da região que corresponde ao fornecimento de recursos não renováveis e provenientes da economia ao sistema.

\section{Breve histórico sobre a geração de energia}

Por volta do século XVIII, através da Revolução Industrial onde que houve um desenvolvimento tecnológico através do carvão como principal fonte de energia, veio também a concepção de que a natureza seria uma fonte de ilimitada de recursos à disposição do homem (Hemery et. al., 1993). Com a descoberta do petróleo, o carvão deixa de ser a principal fonte de energia No século $X X$, o uso intensificado do petróleo impulsionou ainda mais o processo de industrialização e os desenvolvimentos econômicos, ocasionando intensa modificação do espaço ocupado pelo homem (Santos, 1977).

Já o do uso da geração de eletricidade impulsionou os avanços tecnológicos e criou uma forte dependência dos combustíveis fósseis para geração de energia, a exemplo do carvão mineral. (Strapasson, 2004). Atualmente, o consumo de energia elétrica vem crescendo a cada dia mais, tanto no setor industrial, comercial ou residencial. Se esse aumento continuar de forma significativa pode ser que venha ocorrer uma crise devido à escassez energética. Segundo Goldemberg (1998), esse consumo de energia vem crescendo em média $2 \%$, ao ano na última década com perspectiva de que venha a duplicar em 30 anos se esse consumo de energia elétrica continuar dessa forma. Em específico, no Brasil isso significa uma rápida exaustão de reservas de combustíveis fósseis.

Segundo Braga (2002), fontes renováveis são os recursos naturais que depois de utilizados ficam disponíveis novamente naturalmente através de ciclos que ocorrem na natureza, ou seja, sua taxa de uso é menor que a taxa de regeneração. Atualmente, o desenvolvimento sustentável é visto como uma necessidade global, para que as gerações vindouras tenham condições de sobrevivência (Brundtland, 1987). Apesar do Brasil não estar entre os países desenvolvidos estabelecidos no protocolo de Kyoto em 1992, é necessário seguir o princípio de responsabilidade comum, pois é uma questão que diz respeito à todas nações. A necessidade de utilização de fontes de energias renováveis é de grande importância, uma vez que contribuem para a mitigação das emissões de gases de efeito estufa (GEE) (Udaeta, 1997).

A princípio as usinas elétricas utilizavam o carvão mineral como combustível, sendo substituído por outros combustíveis fósseis como, por exemplo, energia nuclear. Existem usinas hidrelétricas que obtém a energia través do acionamento de turbinas pela queda d'água sendo este um sistema de geração de longa vida útil e renovável, podendo acarretar conseqüências ao meio ambiente como perda da biodiversidade.
Fontes renováveis como a energia eólica também são utilizadas, mas, em pequena escala, sendo obtida através da energia cinética dos ventos onde que, as células de combustível, que combinam eletroquimicamente hidrogênio e oxigênio para produzir eletricidade, energia geotérmica, como também à conversão solar direta em eletricidade, através de um sistema chamado fotovoltaico (sistema constituído de um conjunto de painéis para a captação de energia solar). Segundo estimativas de Hall (1991), a utilização de um terço dos resíduos disponíveis seria capaz de atender a $10 \%$ do consumo elétrico mundial e com a implantação de um programa de plantio de 100 milhões de hectares de culturas especialmente para esta atividade, seria possível atender $30 \%$ do consumo.

\section{Descrição dos Sistemas \\ Aterro Sanitário}

O aterro sanitário utilizado como base para este trabalho está localizado na cidade de São Paulo, opera com a carga anual de 1,70 toneladas de resíduos sólidos.

\section{ETE}

A estação de tratamento de esgoto situa-se na cidade de Uppsala, Suécia, que trata de $100.000 \mathrm{~m}^{3}$ de esgoto anualmente pelo método de Lodo Ativado. De acordo com o protocolo de Kyoto (MRE, 1997), que estabelece que até 2010 o uso de fontes de energia renovável deveria alcançar $10 \%$ da matriz energética (Braga et al, 2002),

\section{Usina autônoma}

A usina autônoma é idealizada para o Estado de São Paulo em uma área de 4.360 ha, onde 1.494 ha seriam utilizados para o plantio de cana, produzindo 40.000 litros de álcool diários, e 27,1 kWh de eletricidade excedente por tonelada de cana utilizando uma caldeira de 67 bar $/ 480^{\circ} \mathrm{C}$, operando durante 210 dias ao ano.

\section{REVISÃO BIBLIOGRÁFICA}

A revisão bibliográfica está dividida em duas partes, a primeira trata de apresentar as bibliografias utilizadas como base de dados para este trabalho, a segunda parte apresenta as bibliografias que utilizaram a pegada ecológica modificada (Zhao et al, 2004)

Primeira parte, para a realização dos cálculos necessários para a elaboração deste trabalho, foram utilizados as bibliografias apresentadas abaixo:

Frimaio (2011) descreve que devemos considerar que a queima do biogás gerado no aterro sanitário, além de gerar eletricidade, também transforma o metano em dióxido de carbono e vapor d'água, e seu aproveitamento deve ser considerado, uma vez que num período de 100 anos, 1 grama de metano contribuiria 21 vezes mais para a formação do efeito estufa do que 1 grama 
da dióxido de carbono (World Bank, 2004).

Frimaio (2011) realizou a contabilidade em emergia do Aterro Sanitário São João, dessa forma foi possível estimar todos os fluxos de energia que o sistema utiliza, assim sendo, alguns insumos foram contabilizados, como por exemplo, maquinários e materiais de construção. Os fluxos de energia do sistema foram transformados para uma métrica comum, o joule de emergia solar, através da multiplicação das quantidades de cada insumo pela sua respectiva transformidade

Corsini et. al (2011), concluem que através do indicador ELR verificou-se que o resultado obtido pelo sistema da usina autônoma representa que a energia solar dos recursos não renováveis e dos recursos advindos do sistema econômico é 2,12 vezes maior que a energia solar equivalente dos recursos renováveis utilizados, indicando uma baixa carga ambiental e um baixo estresse ambiental. Devido ao resultado obtido no índice de sustentabilidade, fica clara a necessidade de melhorias com o intuito de ter um aumento na sustentabilidade do ciclo.

Bjoklund et al., (2001), realizaram o estudo em uma Estação de Tratamento de Esgoto (ETE) verificando que o sistema apresenta poucas fontes de energia renovável, seu índice é de 0,0657\%, sendo inferior ao mínimo de $10 \%$ estabelecido pelo protocolo de Kyoto (MRE, 1997). Com relação ao índice de sustentabilidade, o valor obtido é muito baixo, com isso é necessário que haja grandes melhorias para que ocorra aumento na sustentabilidade do ciclo.

Segunda parte, aqui são apresentadas as bibliografias de trabalhos que utilizaram a pegada ecológica modificada como métrica.

Odum (1996) desenvolveu e aplicou a metodologia em emergia para o cálculo de sistemas ambientais, processos industriais e serviços. Utilizando a síntese emergética Odum, (1996) e Zhao et al, (2004) propuseram um cálculo de Pegada Ecológica (PE) e Capacidade de Carga (Cc) modificadas, sendo assim a Pegada Ecológica modificada é considerada como a área da região que corresponde ao fornecimento de recursos não renováveis e provenientes da economia. É considerada a capacidade de carga, a área necessária para o fornecimento de recursos renováveis ao sistemas. A diferença entre os dois valores (Cc-PE), mostrará se o sistema está em débito ou em crédito com a biosfera.

Björklund et al. (2001), calcularam a transformidade da energia elétrica gerada através do aproveitamento do biogás produzido por um biodigestor instalado na estação de tratamento de esgoto com sistema de tratamento convencional, na Suécia. Comparando o valor obtido com a transformidade da energia elétrica utilizada no sistema, que é composta por uma parte de energia elétrica de usina nuclear e outra parte por energia elétrica de hidroelétrica, concluíram que se o sistema fosse utilizado para geração de energia elétrica, seria ineficiente, pois a transformidade encontrada é maior do que as de outras fontes de energia elétrica.

Silva (2006) fez a comparação entre dois sistemas, o primeiro utiliza um sistema de biodigestão no Estado do Rio de Janeiro e o segundo opera com o sistema de Lodo Ativado na Estação de Tratamento de Efluentes no Estado de São Paulo. Foram quantificadas as emergias envolvidas nestes sistemas e comparado os dois modelos de tratamento utilizando indicadores ambientais. Comparando esses dois sistemas pode-se verificar que o sistema de Lodo Ativado apresentou melhor performance com relação à Capacidade de Carga e à Pegada Ecológica. Quando calculado e diferença entre esses dois indicadores observou-se que o Biodigestor obteve um valor negativo, apresentando um déficit de área.

\section{OBJETIVOS}

\section{Objetivo Geral}

O presente artigo tem como objetivo realizar o cálculo da Pegada Ecológica modificada dos sistemas propostos, que utilizaram a Contabilidade em emergia e compará-las. O primeiro trata da Geração de Energia Instalado em uma Usina Autônoma no Estado de São Paulo, o segundo em uma Estação de Tratamento de Esgoto (ETE) situada em Uppsala, Suécia e o terceiro sistema é sobre o Aproveitamento Energético dos Resíduos Sólidos Urbanos em Aterro Sanitário no Estado de São Paulo.

\section{Objetivos Específicos}

Apresentar o resultado dos sistemas estudados, determinando o superávit ou déficit de área de cada sistema.

\section{METODOLOGIA}

A pegada ecológica modificada estabelece uma relação entre a emergia e pegada ecológica, desenvolvida por Rees e Wackernagel (1994), associa os recursos utilizados por um sistema à área necessária para o suprimento destes recursos.

Zhao et al. (2004) associam os fluxos de emergia, que também representam os recursos utilizados por um sistema, a uma área. Desta forma, propõem um cálculo de pegada ecológica modificada, que leva em conta os recursos utilizados pelo sistema $(\mathrm{N}+\mathrm{F}+\mathrm{R})$ em relação à densidade emergética local. Zhao et al (2004) propõem ainda o cálculo da Capacidade de Carga modificada $(\mathrm{Cc})$, que leva em conta a emergia renovável do sistema e a densidade emergética do planeta. Quanto maior o valor de Cc, ou seja, quanto maior for o fluxo de emergia renovável utilizada pelo sistema, maior será o tempo em que o ambiente poderá suportar este sistema.

A Pegada Ecológica é calculada através da razão entre os recursos não renováveis somado com os 
recursos pagos, dividido pela emergia da região sobre a área da região conforme equação 1.

A Capacidade de Carga é dado pelo razão entre os recursos renováveis divididos pela emergia da Terra pela área da Terra, de acordo com a equação 2 .

A diferença entre Cc e PE indica um débito (custo ao planeta) ou crédito (benefício ao planeta) do sistema para com o meio ambiente, é calculada

$$
\begin{aligned}
& \mathrm{PE}=\frac{(\mathrm{N}+\mathrm{F})}{\mathrm{Em}_{\mathrm{p}} / A_{P}} \\
& \mathrm{Cc}_{\mathrm{c}}=\frac{\mathrm{R}}{\mathrm{Em}_{\mathrm{T}} / \mathrm{A}_{\mathrm{T}}} \\
& \mathrm{Cc}-\mathrm{PE}=\frac{\mathrm{A}_{\mathrm{T}}}{\mathrm{Em}_{\mathrm{T}}} \mathrm{x}\left(\mathrm{R}-\frac{\mathrm{g}}{\mathrm{y}} \mathrm{x}(\mathrm{N}+\mathrm{F})\right)
\end{aligned}
$$

pela razão entre a área da Terra pela emergia da Terra, multiplicado pela diferença entre os recursos renováveis e $g$ representada na equação 4 , multiplicado

pela somatória dos recursos não renováveis e os recursos pagos.

\section{Equação 1}

Equação 2

Equação 3
$\mathrm{O}$ valor de EmT refere-se à emergia total da Terra e AT é a área da Terra, sendo $\mathrm{R}$ a emergia renovável utilizada pelo sistema, $\underline{\underline{G}}$ é a razão entre a área da região sobre a área $\mathrm{Y}$ 「erra estudada, dividido pela razão entre a emergia da região pela emergia da Terra. EmP é ao total de emergia da região em que está o sistema e AP é a área desta região, conforme equação 4 .

$$
\mathrm{g} / \mathrm{y}=(\mathrm{Amp} / \mathrm{At}) /(\mathrm{Emp} / \mathrm{Emt})
$$

Equação 4

Tabela 1 . Avaliação da Emergia da Estação de Tratamento de Esgoto em Uppsala, Suécia. (adaptado de Björklund et al., 2001)

\begin{tabular}{llcl}
\hline & & Emergia & $\%$ \\
& Descrição & $/$ (sej/ano) & $/(\mathrm{sej} / \mathrm{sej})$ \\
\hline & & & \\
2 & Recursos Renováveis & $1,0.10^{14}$ & $<1 \%$ \\
3 & Recursos não renováveis & $4,24.10^{16}$ & $27,8 \%$ \\
& Recursos Pagos & $1,097.10^{17}$ & $71,8 \%$ \\
\hline & Emergia Total com os serviços do ambiente & $152.10^{15}$ & $100 \%$ \\
\hline
\end{tabular}


Tabela 2 . Avaliação da Emergia da Usina Autonoma, São Paulo. (adaptado de Silva, 2009)

\begin{tabular}{|c|c|c|c|}
\hline$\frac{\pi}{\circ}$ & Descrição & $\begin{array}{l}\text { Emergia } \\
\text { /(sej/ano) }\end{array}$ & $\begin{array}{l}\% \\
/ \text { (sej/sej) }\end{array}$ \\
\hline 1 & Recursos Renováveis & $3,38.10^{18}$ & $32 \%$ \\
\hline 2 & Recursos não renováveis & $4,54.10^{17}$ & $8 \%$ \\
\hline 3 & Recursos Pagos & $1,80.10^{18}$ & $60 \%$ \\
\hline & Emergia Total com os serviços do ambiente & $5,63.10^{18}$ & $100 \%$ \\
\hline
\end{tabular}

Tabela 3 . Avaliação da Emergia de Aproveitamento Energético dos Resíduos Sólidos Urbanos em Aterro Sanitário, São Paulo. (adaptado de Frimaio, 2011)

\begin{tabular}{llll}
\hline & & Emergia & $\%$ \\
\hline \multicolumn{2}{c}{ Descrição } & /(sej/ano) & (sej/sej) \\
\hline & & & \\
2 & Recursos Renováveis & $4,14.10^{19}$ & $34,5 \%$ \\
3 & Recursos não renováveis & $1,39.10^{18}$ & $1,0 \%$ \\
& Recursos Pagos & $7,74.10^{19}$ & $64,5 \%$ \\
\hline & Emergia Total com os serviços do ambiente & $1,20.10^{20}$ & $100 \%$
\end{tabular}

Os resultados obtidos com o uso da metodologia utilizando as equações 1, 2 e 3, são apresentadas na tabela 4. 
Tabela 4. Cálculo da PE e da Cc dos sistemas da estação de tratamento de esgoto (ETE), usina autônoma e aproveitamento de resíduos sólidos urbanos em uma aterro, segundo a proposta de Zhao et al., 2004(*).

\begin{tabular}{lccccc}
\hline & $\begin{array}{c}\mathrm{R} \\
/(\mathrm{sej})\end{array}$ & $\begin{array}{c}\mathrm{N}+\mathrm{F}) \\
/(\mathrm{sej})\end{array}$ & $\begin{array}{c}\mathrm{PC} \\
/\left(\mathrm{m}^{2}\right)\end{array}$ & $\begin{array}{c}/\left(\mathrm{m}^{2}\right) \\
(\mathrm{Cc}-\mathrm{PE})\end{array}$ & $/\left(\mathrm{m}^{2}\right)$ \\
\hline ETE & $1,0.10^{14}$ & $1,521.10^{17}$ & $3,22.10^{3}$ & $2,86.10^{-4}$ & $3,22.10^{3}$ \\
& & & & & \\
& & & & & \\
Usina autônoma & $3,38.10^{18}$ & $3,834.10^{18}$ & $1,09.10^{8}$ & $6,96.10^{6}$ & $1,02.10^{8}$ \\
& & & & & \\
$\begin{array}{l}\text { Aproveitamento de } \\
\text { resíduos }\end{array}$ & $7,741.10^{19}$ & $7,88.10^{19}$ & $1,33.10^{9}$ & $2,43.10^{8}$ & $1,0 p \cdot 10^{9}$
\end{tabular}

$\left({ }^{*}\right)$ Cálculos detalhados no anexo.

Verifica-se que o resultado obtido no sistema de Aproveitamento de Resíduos com relação à Pegada Ecológica é 34,91 vezes maior que o valor do sistema de Usina Autônoma e 2.433.1010 vezes maior que a Estação de Tratamento de Efluentes, dessa forma o sistema com a menor pegada ecológica é a Estação de Tratamento de Efluentes. Indicando que a Usina autônoma é o sistema que necessita de maior área para implementação.

Com relação à Capacidade de Carga o maior valor obtido foi o do Sistema de Aproveitamento de Resíduos, sendo ele 12,20 vezes maior que a Usina Autônoma e 3,39.1014 vezes maior que o sistema de Aproveitamento de Resíduos. Indicando que, sendo o maior fluxo de emergia renovável pelo sistema, maior será o tempo em que o ambiente poderá suportar este sistema.

Verifica-se que o resultado da diferença entre a Capacidade de Carga e a Pegada Ecológica foi melhor na Estação de Tratamento de Esgoto, sendo que este é 3,17.1003 menor que a Usina Autônoma e 3,39.1005 vezes menor que o sistema de Aproveitamento de Resíduos.

\section{CONCLUSÕES}

Foram realizados o cálculo da Pegada Ecológica modificada, da Capacidade de Carga, e a diferença entre esses indicadores. A partir dos resultados determinados, verifica-se que o sistema de produção de energia elétrica com Aproveitamento de resíduos apresenta melhor desempenho, pois a área excedente segundo a metodologia aplicada é maior, sendo assim o "rastro" deixado no planeta por esse sistema é bem inferior comparado aos sistemas discutidos.

Para que os sistemas de geração de energia elétrica na ETE e na Usina Autônoma possam apresentar melhor desempenho, seria necessária a substituição de alguns fluxos de energia de fontes pagas por recursos de fontes renováveis.

\section{REFERÊNCIAS BIBLIOGRÁFICAS}

BJÖRKLUND, J.; GEBER, U; RYDBERG, T. (2001) Emergy analysis of municipal wastewater treatment and generation of electricity by digestion of sewage sludge, Resources, Conservation and Recycling, 31, p.293-316.

BRAGA,B.; HESPANHOL,I.; CONEJO,J.G.L; BARROS,M.T.L; SPENCER,M.; PORTO,M.; NUCCI,N.; JULIANO,N.; EIGER,S. (2002) - Introdução à Engenharia Ambiental, São Paulo, Prendtice Hall

BRUNDTLAND, G. H. - Our common future: World Commission on Environment and Development. Oxford: Oxford University Press, 1987.

CORSINI, I.; CARVALHO, B. S.; PEREIRA, E. M.; CUNHA, M. C. A.; SILVA, C. C. (2011): Contabilidade em emergia de dois sistemas de geração de energia elétrica com utilização de resíduos - Revista Agrogeoambiental, Inconfidentes v. 03, n. 01, p. 83-91.

FOLKE, M., C.; COSTANZA, R. (Eds.), Investing in Natural Capital. Island Press, Washington, DC, p. 362390.

FRIMAIO, G; ALMEIDA,C; BIAGIO, F; BONILLA, S.2011 Aproveitamento Energético dos Resíduos Sólidos Urbanos em Aterro Sanitário

GOLDEMBERG, J. Energia, meio ambiente e desenvolvimento. São Paulo: Edusp, 1998. 
HALL, D.O. Biomass Energy. Energy Policy, v. 19, n. 8, p. 711-737, Oct. 1991.

HEMERY, D.; DEBEIR, J.; DELÉAGE, J. Uma história da energia. Brasília: Editora da Universidade de Brasília, 1986.Intergovernmental Panel on Climate Change (IPCC, 2006)

ODUM, H.T. - Environmental accounting - Emergy and environmental decision making, Ed. John Wiley \& Songs 1996. p.370.

ODUM, H.T. Emergy evaluation of an OTEC electrical power system. Energy v.25, p.3989-3993, 2000

SILVA, 2006 - Estudo de Caso de Sistemas de Tratamento de Efluentes Domésticos com o Uso de Indicadores Ambientais

SILVA, 2009 - A Atribuição de Custos em Sistemas Energéticos Agropecuários: Uma Análise em Emergia, Termoeconomia e Economia

SANTOS, M. Técnica, espaço, tempo: globalização e meio técnico-científico informacional. 3.ed. São Paulo: Hucitec., 1977. 190p.

STRAPASSON, Alexandre Betinardi. A energia térmica e o paradoxo da eficiência energética-desafios para um novo modelo de planejamento energético. 2004. 134p. Dissertação (Mestrado em Energia ) - Programa Interunidades de Pós-Graduação em Energia. São Paulo, 2004.

REES, W.E.; WACKERNAGEL, M.; (1994) - Ecological footprints and appropriated carrying capacity: measuring the natural capital requirements of the human economy. In: JANSSON, A.-M., HAMMER,

UDAETA, M.E.M. Planejamento integrado de recursos energéticos -PIR para o setor elétrico (pensando o desenvolvimento sustentável). 1997. 347p.Tese (Doutorado)- Escola Politécnica da Universidade de São Paulo, São Paulo, 1997.

ZHAO, S.; LIB, Z.; LIA, W. (2004). A modified method of ecological footprint calculation and its application, Ecological Modeling, 185, p. 65-75. 